\title{
Maestras de preescolar: características reconocidas en niños y niñas con aptitudes sobresalientes
}

\author{
Rosa Adriana Morales-Nava, Lic. ${ }^{a}$ \\ Escuela Normal Particular Berta von Glümer, \\ Secretaría de Educación Pública, México \\ Mercedes Rosalía González-Arreola, Mg. ${ }^{b}$ \\ Universidad Nacional Autónoma de México, México \\ Blanca Ivet Chavez-Soto, Ph. D. ${ }^{c}$ \\ Universidad Nacional Autónoma de México, \\ Escuela Normal Particular Berta von Glümer, México
}

fadrianamorana@gmail.com

\section{Resumen (analítico)}

El presente trabajo tuvo como objetivo identificar las características que las profesoras de preescolar reconocían en las niñas y los niños con aptitudes sobresalientes. Es una investigación cuantitativa, transversal y exploratoria. Participaron 58 profesoras de preescolar. Se empleó la técnica de redes semánticas naturales modificadas a través de cuatro preguntas estímulo: qué características tienen los niños con aptitud sobresaliente en el área cognitiva, en el área creativa, en el área psicomotriz y área socioemocional. Los resultados mostraron que las profesoras lograron reconocer algunos rasgos de los niños sobresalientes que coinciden con lo reportado en la literatura, lo que sugiere que ellas pueden ser un elemento clave en la detección de la población si se les proporcionan los instrumentos apropiados con las características del alumnado sobresaliente.

\section{Palabras clave}

Red semántica, aptitud sobresaliente, preescolar, inteligencia, creatividad.

\section{Thesauro}

Tesauro de Ciencias Sociales de la Unesco.

\section{Para citar este artículo}

Morales-Nava, R. A., González-Arreola, M. R., \& Chávez-Soto, B. I. (2021). Maestras de preescolar: características reconocidas en niños y niñas con aptitudes sobresalientes. Revista Latinoamericana de Ciencias Sociales, Niñez y Juventud, 19(3), 1-21. https://dx.doi.org/10.11600/rlcsnj.19.3.5066

\section{Historial}

Recibido: 23.06 .2021

Aceptado: 02.02.2021

Publicado: 31.08 .2021

\section{Información artículo}

Este artículo presenta los resultados de la investigación desarrollada de enero a noviembre de 2020. Área: ciencias sociales; subárea: educación. 


\section{Preschool teachers: recognized characteristics in gifted children}

Abstract (analytical)

This study had the objective of identifying characteristics that preschool teachers recognize in gifted boys and girls through a quantitative, cross-sectional and exploratory investigation. A total of 58 preschool teachers participated in the research. A modified natural semantic networks technique was used by asking four stimulus questions: What characteristics do gifted children have in the cognitive area?; in the creative area?; in the psychomotor area; and in the socio-emotional area. The results showed that the teachers were able to recognize some characteristics of gifted children that coincide with what is reported in scientific literature. This suggests that they may be a key element in detecting the population of gifted children if they are provided with the appropriate instruments outlining the characteristics of outstanding students.

Keywords

Semantic network, outstanding aptitude, preschool, intelligence, creativity.

\section{Professores de pré-escolar: características reconhecidas em meninos e meninas excepcionais}

Resumo (analítico)

O presente trabalho teve como objetivo identificar ases características que professores de pré-escola reconhecem em meninos e meninas com habilidades destacadas. É uma investigação quantitativa, transversal e exploratória. Participaram 58 professores de pré-escola, foi utilizada a técnica de redes semânticas naturais modificadas por meio de quatro questões-estímulo: Quais são as características das crianças com aptidão destacada na área cognitiva, na área criativa, na área psicomotora e na área socioemocional. Os resultados mostraram que os professores conseguiram reconhecer algumas características das crianças destacadas que coincidem com o que foi relatado na literatura, o que sugere que podem ser um elemento fundamental no detecção da população se lhes forem fornecidos os instrumentos adequados com as características dos alunos destacados.

Palavras-chave

Rede Semântica, Aptidão Excepcional, Pré-escola, Inteligência, Criatividade.

Información autores

[a] Secretaría de Educación Pública, Estado de México, México. Licenciada en Educación Preescolar, Normal Particular Berta Von Glümer. (iD) 0000-0001-9322-3278. H5: 0. Correo electrónico: fadrianamorana@gmail.com

[b] Universidad Nacional Autónoma de México, Ciudad de México, México. Licenciada en Psicología, Universidad Autónoma de Sinaloa. Maestra en Psicología con Residencia en Educación Especial, Universidad Nacional Autónoma de México. (iD 0000-0001-8811-4974. H5: 0. Correo electrónico: meroga_agorem@hotmail.com

[c] Universidad Nacional Autónoma de México, Ciudad de México, México. Licenciada en Psicología, Universidad Nacional Autónoma de México. Maestra en Psicología con Residencia en Educación Especial, Universidad Nacional Autónoma de México. Doctora en Psicología Educativa y del Desarrollo de la Universidad Nacional Autónoma de México. (ID) 0000-0001-5922-2351. H5: 3. Correo electrónico: mil_chavez@hotmail.co 


\section{Introducción}

n punto medular en las escuelas es la participación de los docentes en la iden-
tificación y atención de los niños y niñas con necesidades específicas (Valadez et al., 2019). Por esta razón, se reconoce la importancia de que los y las profesoras estén capacitados con el propósito de lograr una inclusión efectiva, al dar oportunidad a todas y todos los estudiantes para que participen en los ámbitos familiar, social y laboral; tal y como se establece en la Ley General de Educación al señalar que se debe brindar educación a las personas independientemente de sus condiciones lingüísticas, culturales, necesidades educativas especiales o aptitudes sobresalientes (Diario Oficial de la Federación, 2011).

Por otro lado, dentro del contexto escolar resulta evidente que aun cuando se ha intentado brindar atención a los niños y niñas sobresalientes, en ocasiones las acciones son insuficientes, al considerar que ellos no requieren de una educación especial porque se tiene la creencia de que su desarrollo y aprendizaje se consolida de forma gradual (Covarrubias \& Marín, 2015; Chávez-Soto \& Acle, 2018). Asimismo, se ha encontrado que las y los estudiantes sobresalientes son un grupo heterogéneo, ya que, así como existen niños y niñas con comportamientos aceptables en las aulas, también coexisten las capacidades sobresalientes en alumnos y alumnas con conductas disruptivas (Department of Elementary \& Secondary Education, 2019; Renzulli, 2012; Sternberg et al., 2010). Además, la evidencia empírica ha demostrado que las y los estudiantes sobresalientes, al aprender más rápido los contenidos académicos, suelen ser etiquetados como problema, debido a que en ocasiones se distraen, son inquietos y consideran que las tareas escolares son aburridas. De ahí la necesidad de identificarlos para proporcionarles actividades motivantes y adecuadas a sus intereses (González \& Chávez-Soto, 2021; Palacios, 2018).

En cuanto a la incidencia de alumnos y alumnas sobresalientes, existen datos en México que refieren que durante el ciclo escolar 2015-2016 se atendieron a 39709 alumnos sobresalientes de primaria, lo que representa el 1.42\% de la población (Senado de la República, 2016) y para la 2019-2020 la cifra decrementó a 20690 (Secretaría de Educación 
Pública [SEP], 2020). Se debe mencionar que estos porcentajes están muy por debajo de lo reportado en otros estudios, en los cuales se observa que en las escuelas existe entre un $5 \%$ y un $15 \%$ de niños y niñas sobresalientes (Chávez-Soto, 20o8; Renzulli, 2012). A su vez, ello hace reflexionar sobre si los mecanismos empleados por el Gobierno son los más adecuados para detectar a la población de estudiantes sobresalientes o si existen otros factores que influyen en el proceso. Al respecto, Covarrubias (2018) indica que estas diferencias en los porcentajes se deben al concepto retomado para la identificación; por ejemplo, si se utiliza el término de superdotado que hace referencia aquellos individuos con un coeficiente intelectual superior a 130, concepción basada exclusivamente en la medición psicométrica de la inteligencia al considerarla una capacidad innata. En este caso la cantidad de individuos detectados es alrededor del 1\% y 3\% (Enríquez \& Arredondo, 2018). Pero si se retoma un término más flexible, como lo es alumnos y alumnas con aptitud sobresaliente, la incidencia aumenta hasta un $20 \%$.

Es pertinente mencionar que en México se emplea el concepto de alumnos y alumnas con aptitudes sobresalientes para referirse a un amplio grupo de individuos que destacan principalmente en cinco áreas: intelectual, creativa, socioafectiva, psicomotriz y artística. Se debe mencionar que desde el punto de vista sociocultural el desarrollo de las aptitudes sobresalientes se deriva de un contexto social facilitador; de ahí radica la importancia de identificar a los niños y niñas tempranamente (SEP, 2006).

Para lograr una detección adecuada de los estudiantes sobresalientes la SEP elaboró la propuesta de intervención Atención educativa a alumnos y alumnas con aptitudes sobresalientes (SEP, 2006) y en los Lineamientos de la acreditación, promoción y certificación anticipada de las y los alumnos sobresalientes de educación básica (SEP, 2011). Además, uno de los principales avances fue la siguiente definición:

Los niños sobresalientes son aquellos que destacan significativamente del grupo social y educativo al que pertenecen en uno o más de los siguientes campos: científico-tecnológico, humanístico-social, artístico o de acción motriz, como esta población presenta necesidades especificas, requieren de un contexto facilitador para desarrollar sus capacidades. (SEP, 2006, p. 59)

Otro logro importante, es la Propuesta de promoción anticipada (SEP, 2011) que es una estrategia educativa denominada «aceleración» y da la posibilidad a que las y los estudiantes ingresen antes a la escuela (admisión temprana). También, se propuso la omisión de un grado escolar sin cambiar de nivel educativo con el propósito de que la niña o niño dejen de 
cursar el grado escolar inmediato que les corresponde de acuerdo con su edad cronológica e iniciar el ciclo escolar en el grado superior siguiente.

Por lo tanto, se otorga un papel preponderante a las y los docentes —desde preescolar hasta secundaria - para seleccionar a las y los alumnos con aptitudes sobresalientes; por lo que es necesario que reconozcan las principales características de los sobresalientes para lograr una preidentificación eficaz y brindarles el apoyo educativo acorde con sus necesidades. Al respecto, surge la inquietud por saber si el contar solo con estos documentos es suficiente para que los docentes conozcan los rasgos que la literatura y la evidencia empírica mencionan de dicha población. En cuanto a las características vinculadas con las y los estudiantes sobresalientes, estas han cambiado con el tiempo y dependen de la cultura; pero en la literatura se han agrupado en cuatro dimensiones (Gargiulo, 2011): habilidades cognitivas, creativas, motrices y socioemocionales, las cuales se mencionan a continuación.

Los investigadores reconocen que una de las características más comunes de las y los alumnos sobresalientes es que aprenden a un ritmo más rápido, así como poseen una mayor profundidad y extensión en el aprendizaje (Chávez-Soto, 2014; Chávez-Soto et al., 2014; Valadez, et al., 2005); otros encontraron que sus periodos de atención son más largos cuando se abordan temas de su interés, se destacan en la imaginación y les gusta asumir riesgos (Rayo, 2001). Para Gargiulo (2011) estos niños y niñas presentan rapidez y facilidad para aprender, destreza superior para resolver problemas, amplio vocabulario, comprensión de ideas complejas y abstractas. Por ello, uno de los criterios más empleados para detectar a las y los alumnos sobresalientes es el empleo de pruebas de inteligencia que permiten reconocer los altos coeficientes intelectuales (De Zubiría, 2011), que se vinculan con un elevado nivel de pensamiento abstracto, razonamiento verbal y numérico, relaciones espaciales, memoria, fluidez, procesamiento y recuperación selectiva de la información (Lorenzo, 2006).

Por otro lado, Gargiulo (2011) y Renzulli (2011) reconocen que la elevada creatividad es otra característica de las y los estudiantes sobresalientes. El estudio de esta variable inició con Guilford en 1950 al establecer las características del pensamiento creador (Chacón, 2005; González, 2004). Posteriormente, Torrance (1977) reconoció que la era espacial provocó cambios en la visión de la creatividad y proliferaron las opiniones sobre el tema. Rodríguez et al., (2015) indicaron que la creatividad es la capacidad para resolver problemas de manera automática, productiva y original. Para Torrance (2008), la creatividad 
es un proceso que se expresa en los cambios que se descubren, en los nuevos elementos y en las relaciones; además, la operacionalizó a través de los siguiente indicadores:

- Fluidez: capacidad para producir un gran número de ideas.

- Flexibilidad: aptitud para cambiar de una línea de pensamiento a otra.

- Originalidad: habilidad para aportar ideas o soluciones que están lejos de lo obvio.

- Elaboración: destreza para llenar con detalles las creaciones.

En el caso de los estudiantes sobresalientes es común encontrar puntuaciones elevadas en la creatividad; esta se percibe en las niñas y niños a través de la curiosidad, la capacidad para solucionar problemas, gusto por asumir riesgos, comprensión de ideas complejas y por su imaginación (Chávez-Soto et al., 2014; Metin \& Aral, 2020; Valadez et al., 2005). Al respecto, la SEP (2006) menciona que las y los alumnos con aptitudes sobresalientes presentan habilidad para producir un gran número de ideas diferentes, lo que se concreta en productos novedosos como respuesta a las situaciones y problemas del contexto social del individuo.

Distintos autores han mencionado que las niñas y niños sobresalientes se desarrollan a un ritmo más rápido en el área psicomotriz en comparación con sus pares; por ejemplo, se ha encontrado que, en algunas ocasiones, ellos sostienen la cabeza en los primeros días del nacimiento, gatean y se paran alrededor de los seis meses, caminan a los nueve meses, recortan con las tijeras a los dos años, escriben aproximadamente a los tres años, andan en bicicleta, patines o saltan la cuerda a los cuatro años (Bildiren, 2018; Metin \& Aral, 2020).

Por otro lado, Gagné (2012), Metin y Aral (2020), SEP (2006) y Slater y Howitt (2018) mencionan que la aptitud psicomotriz se refiere a la habilidad para emplear el cuerpo de forma muy diferenciada con propósitos expresivos y para el logro de las metas. Además, implica el empleo de las siguientes habilidades:

- Físico-motrices: destaca la vigorización física, fuerza, resistencia, velocidad, coordinación, flexibilidad y capacidad para mantener el control de su propio cuerpo.

- Cognoscitivas: relacionadas con la capacidad de planeación, supervisión, autocorrección y otros procesos implicados en la regulación del comportamiento para cumplir con las metas.

- Afectivo-sociales: destrezas de colaboración, interacción y juego que desempeñan un papel primordial para el desarrollo de habilidades personales y de trabajo en equipo. 
La SEP (2006) enfatiza que las habilidades psicomotrices se relacionan entre sí de forma diferente y dependen en gran medida de la actividad que el individuo desea desarrollar. $\mathrm{Al}$ respecto, Gardner (2001) señala que estas habilidades forman parte del repertorio de las habilidades cognitivas, por lo que la capacidad intelectual juega un papel importante en la realización y optimización del movimiento del cuerpo.

Uno de los aspectos dentro del ámbito afectivo y social considerado como relevante en las y los estudiantes con altos niveles intelectuales es que ellos son más sensibles e intensos (Piirto \& Fraas, 2012). Por su parte, Karabulut y Ömeroğlu (2021) reconocieron otras características como la profunda preocupación en cuanto a la moralidad y la justicia; desde edades muy tempranas hacen preguntas desconcertantes; son idealistas, perfeccionistas y prefieren trabajar de manera independiente. Con base en lo anterior, Gargiulo (2011), López (2008) y Pfeiffer (2008), Slater y Howitt (2018) consideran que las y los alumnos con aptitud sobresaliente, no solo piensan de forma diferente, sino que además actúan y sienten de manera distinta.

En cuanto a la aptitud social y la interacción, se ha observado que estos estudiantes muestran capacidad para asumir las perspectivas de los otros, razonamiento ético, sensibilidad hacia las necesidades de los demás, disfrutan las relaciones sociales, asumen responsabilidades más allá de lo esperado, tienen aceptación social, resuelven los problemas de los otros, audacia e iniciativa, así como muestran una tendencia a influir sobre sus compañeros y dirigir las actividades de grupo (Blanco, 2001; Gargiulo, 2011; López, 2008; Martín, 2004; Pfeiffer, 2008).

Para Gargiulo (2011) y Schuur et al. (2021) algunas de las características de los alumnos sobresalientes en el área socioemocional son las siguientes: les agrada trabajar de manera independiente, tienen un elevado nivel de entusiasmo, exhiben capacidades de liderazgo, se relacionan más fácilmente con personas adultas, son sensibles, empáticos, muestran motivación intrínseca, asumen el riesgo, son perfeccionistas, autocríticos, idealistas, intensos, les agrada la rutina, se autorregulan y presentan un alto locus de control interno.

Es pertinente mencionar que el grupo de estudiantes sobresalientes es heterogéneo, ya que pueden encontrarse niñas y niños que cubran con todos los indicadores o solo con algunos; por este motivo es necesario que los docentes reconozcan sus características, ya que ellos son un punto clave para su identificación. Por lo tanto, desde la postura de esta investigación, las profesoras y profesores cumplen una función importante dentro del ámbito educativo de las y los estudiantes sobresalientes ya que ellos los remiten a diversos programas para favorecer sus potencialidades. Sin embargo, la evidencia empírica ha 
reportado que las percepciones que tienen los docentes sobre la identificación de niños sobresalientes en ocasiones no son tan efectivas (Alonso \& Benito, 2004; Valadez et al., 2005). En cambio, otros investigadores como Endepohls-Ulpe y Ruf (2005), Hong et al. (2009), Manzano et al. (2010) y Zavala (2004) indican que el profesor es una fuente confiable al nominar a sus alumnos porque son capaces de reconocer las capacidades intelectuales y creativas. Por ello, consideramos que el docente es una fuente de información que sigue empleándose para la identificación del alumno sobresaliente, aun cuando no está exento de problemas y actualmente es una cuestión que sigue provocando controversias.

Un asunto que es importante retomar es que la SEP propuso que la detección de las niñas y los niños debe comenzar desde el nivel preescolar, lo cual contrasta con la realidad porque al hacer una revisión de las investigaciones realizadas en México la mayoría se centró en los estudiantes de primaria (Covarrubias, 2012). Se tiene como antecedente la propuesta del Programa estatal de fortalecimiento de la educación especial 2005-2010, sobre la identificación temprana de niños y niñas sobresalientes de preescolar en 25 estados de la República a través de estudios piloto donde se aplicaron algunos instrumentos; pero aún no hay datos precisos de los resultados (Páez \& Valladares, 2015).

Por lo tanto, la identificación temprana de niñas y niños sobresalientes es un tema de atención a nivel nacional ya que, entre más pequeños se detecten, se tiene más posibilidad de desarrollar sus capacidades. Al respecto, se hizo un estudio en el Estado de Chihuahua en colaboración con el Centro Huerta Rey de Valladolid; los investigadores a cargo elaboraron una tabla de desarrollo con las siguientes áreas: motor, lenguaje, cognitivo, ayuda y socialización, la cual se empleó para que los maestros de preescolar seleccionaran a los niños sobresalientes y con los resultados se logró validar el instrumento (Covarrubias, 2012). Ante esta iniciativa, y con el propósito de profundizar en el tema de los niños sobresalientes en edad preescolar, se diseñó el presente trabajo que tuvo como objetivo caracterizar a los niños y niñas con aptitudes sobresalientes a partir de las descripciones realizadas por las docentes de preescolar.

\section{Método}

Se realizó una investigación cuantitativa. El tipo de estudio fue transversal, ya que se aplicaron redes semánticas en un solo momento; y fue de carácter exploratorio porque se examinó un tema poco estudiado (Kerlinger \& Lee, 2002). 


\section{Participantes}

Se empleó un muestreo por conveniencia. Colaboraron 58 maestras de nivel preescolar de los municipios de Ecatepec de Morelos, en el Estado de México. La edad de las participantes osciló entre 23 a 62 años $\left(M_{\text {edad }}=36.59\right)$, con un rango de 1 a 34 años de servicio $\left(\mathrm{M}_{\text {años de servicio }}=9.67\right)$ y una experiencia de 1 a 32 años a cargo de grupos de preescolar $\left(\mathrm{M}_{\text {años frente a grupo }=9.11}\right)$. En cuanto a las características de las docentes, se encontró que 26 de ellas tenían licenciatura en preescolar, seis eran psicólogas, ocho pedagogas, nueve licenciadas en educación, una con licenciatura en educación primaria, otra era psicopedagoga y siete con maestría. Con respecto al tipo de escuela en donde laboraban, se observó que 28 trabajaban en escuelas particulares, 29 en preescolares oficiales y solo una laboraba en ambos tipos de escuela. También, se reportó que 45 docentes eran del turno matutino, 10 del vespertino y tres de ellas laboraban en ambos horarios.

Los criterios de inclusión para el estudio fueron: tener una edad entre 20 y 65 años, que laboraran como maestras de preescolar, con mínimo un año de servicio. Los criterios de exclusión fueron que las participantes no firmaran el consentimiento informado.

\section{Instrumentos}

Se empleó un consentimiento informado, el cual fue firmado por las docentes y en el que se les informó sobre el objetivo del estudio y ellas accedieron a participar. Por otro lado, para conocer las características que las docentes identificaban en los estudiantes sobresalientes, se empleó como técnica las redes semánticas naturales modificadas (Reyes-Lagunes, 1993), que son una estrategia que permite definir un conjunto de palabras; usualmente se emplea para el estudio de los significados o expresiones que son reportados por un grupo social determinado (Figueroa et al., 1981).

El procedimiento para realizar las redes semánticas naturales modificadas consiste en seleccionar las oraciones estímulo de las cuales se quiere el significado; estas se proporcionan a un grupo de personas y se solicita que definan el estímulo mediante un mínimo de cinco ideas (Reyes-Lagunes, 1993). Cuando las personas han escrito la lista de palabras, se solicita que le asignen un número a cada una de ellas de manera jerárquica de acuerdo con la cercanía e importancia que tiene cada idea con la oración. Para este estudio se crearon cuatro preguntas estímulo que fueron elaboradas a partir de la propuesta de la SEP (2006), además se incluyó un distractor, los cuales se mencionan a continuación: 
- ¿Qué características tienen los niños y niñas con aptitud sobresaliente en el área cognitiva?

- ¿Qué características presentan los niños y niñas con aptitud sobresaliente en el área creativa?

- ¿Qué características se observan en los niños y niñas con aptitud sobresaliente en el área psicomotriz?

- ¿Qué características muestran los niños y niñas con aptitud sobresaliente en el área socioemocional?

- Distractor: ¿qué campos de formación académica o áreas de desarrollo personal y social se deben de priorizar en niños y niñas con aptitudes sobresalientes?

\section{Procedimiento}

Para este trabajo se realizó lo siguiente: se buscó información en la SEP sobre la definición de alumnos sobresalientes y el modelo que se ha empleado para la detección de esta población. Después, se elaboraron las redes semánticas a partir de cuatro preguntas y un distractor. Se solicitó a través de un oficio el permiso para realizar el estudio. Una vez que se contó con la aprobación, se contactó a las docentes y se les explicó el objetivo de la investigación, se solicitó que las profesoras firmaran el consentimiento y se aplicaron las redes semánticas naturales en un tiempo promedio de 20 minutos. El análisis de los resultados se realizó con el programa Excel ${ }^{\mathrm{TM}} \mathrm{y}$ se siguieron los pasos propuestos por Reyes-Lagunes (1993) para el tratamiento de los datos: primero se obtuvo el tamaño de la red (TR), el peso semántico (PS) y la distancia semántica cuantitativa (DSC) por cada estímulo. Se seleccionaron las definidoras con mayor PS y se obtuvo el núcleo de la red.

\section{Resultados}

En la pregunta referente a las características tienen los niños con aptitud sobresaliente en el área cognitiva, se encontró que el tamaño de la red se conformó por 99 palabras definidoras y, al realizar la suma de ponderación de la frecuencia por la jerarquización para obtener el peso semántico, se incluyeron en la red 14 ideas, debido a que en la definidora número 15 se ubicó el punto de quiebre donde el PS se vuelve asintótico (tabla 1). 


\section{Tabla 1}

Peso semántico (PS) y distancia semántica (DSC) del conjunto de definidoras de la pregunta ¿qué características tienen los niños con aptitud sobresaliente en el área cognitiva?

\begin{tabular}{lcc}
\hline Definidoras & PS & DSC \\
\hline Facilidad para resolver problemas & 172 & 0 \\
Memorización de cosas u objetos & 109 & 36 \\
Son creativos & 94 & 45 \\
Presta atención en periodos más prolongados & 87 & 49 \\
Lenguaje claro y fluido & 68 & 60 \\
Vocabulario amplio & 60 & 65 \\
Facilidad para aprender conocimientos & 58 & 66 \\
Inquietud por experimentar o saber más & 57 & 66 \\
Reflexivos & 55 & 68 \\
Hacen preguntas constantemente & 54 & 68 \\
Razonamiento lógico & 52 & 69 \\
Participativos & 52 & 69 \\
Aprenden con rapidez y facilidad & 51 & 70 \\
Capacidad para comprender temas complejos para su edad & 47 & 72 \\
\hline
\end{tabular}

Como se observa, en el núcleo se encuentra la expresión «facilidad para resolver problemas», en segundo lugar la de «memorización de cosas u objetos con detalle», en tercer sitio está la palabra creativos. La información encontrada en los datos fue interesante ya que permitió observar que las docentes reconocieron distintas características de las y los estudiantes con aptitud sobresaliente que se han descrito en los modelos y en la literatura que pueden estar presentes en la categoría y que ayudan a la identificación de los niños (Gargiulo, 2011; Rodríguez et al., 2015; SEP, 2006).

En cuanto a la pregunta ¿qué características tienen los niños con aptitud sobresaliente en el área creativa? los resultados mostraron que el tamaño de la red tuvo 106 definidoras y con la suma de ponderación de la frecuencia para obtener el peso semántico se incluyeron en la red ocho oraciones y en la idea número nueve se ubicó el punto de quiebre donde el PS se vuelve asintótico (tabla 2).

En el núcleo del área creativa se encontró que las docentes mencionaron la expresión «más imaginativos», en segundo lugar indicaron que los niños tienden a ser hábiles en cuestiones artísticas. En este sentido, Gargiulo (2011) reconoció que los individuos con aptitudes sobresalientes tienen un pensamiento independiente, exhiben ideas originales de forma oral y escrita, demuestran capacidad para solucionar problemas de dife- 
rente forma, crear e improvisar, tal y como lo refirieron las docentes de preescolar que participaron en este estudio.

\section{Tabla 2}

Peso semántico (PS) y distancia semántica (DSC) del conjunto de definidoras de la pregunta ¿qué características tienen los niños con aptitud sobresaliente en el área creativa?

\begin{tabular}{lcc}
\hline Definidoras & PS & DSC \\
\hline Más imaginativos & 231 & 0 \\
Tienden a ser hábiles en cuestiones artísticas (pintura, danza, música) & 178 & 22 \\
Ideas originales & 161 & 30 \\
Destreza para solucionar conflictos o resolver problemas & 112 & 51 \\
Curiosos & 108 & 53 \\
Sabe trabajar con diversos materiales & 78 & 66 \\
Realizan dibujos detallados (dedicados y cuidadosos) & 74 & 67 \\
Muestran creatividad propia & 74 & 67 \\
\hline
\end{tabular}

Para la pregunta ¿qué características tienen los niños con aptitud sobresaliente en el área motriz? se observaron 125 definidoras en el tamaño de la red y la suma de ponderación de la frecuencia para obtener el peso semántico permitió reconocer que la red se conformó por diez ideas ya que en el número once se encontró el punto de quiebre (tabla 3).

\section{Tabla 3}

Peso semántico (PS) y distancia semántica (DS) del conjunto de definidoras de la pregunta ¿qué características tienen los niños con aptitud sobresaliente en el área motriz?

\begin{tabular}{lcc}
\hline Definidoras & PS & DS \\
\hline Coordinación motriz & 191 & 0 \\
Gusto por actividades deportivas & 69 & 63 \\
Mayor motricidad fina & 67 & 64 \\
Control general de su cuerpo & 53 & 72 \\
Habilidad para emplear su cuerpo en formas diferentes con propósitos & 53 & 72 \\
expresivos & 51 & 73 \\
Gatear, caminar, correr, saltar, lanzar, patear y equilibrio corporal & 50 & 73 \\
Movimientos rápidos al realizar actividad física & 48 & 74 \\
Coordinación motriz gruesa & 46 & 75 \\
Equilibrio & 38 & 80 \\
Conocimiento del cuerpo humano & & \\
\hline
\end{tabular}

En el núcleo se ubicó la expresión «coordinación motriz», mientras que en segundo sitio se reportó el gusto por las actividades artísticas. Al respecto, es interesante retomar 
lo descrito por la SEP (2006) en donde se señaló que la aptitud sobresaliente se expresa en distintas áreas del ser humano como la motriz, al definirla como las habilidades de emplear el cuerpo en formas muy diferenciadas con propósitos expresivos y para el logro de las metas.

Finalmente, se analizó la pregunta ¿qué características tienen los niños con aptitud sobresaliente en el área socioemocional? El análisis de los datos permitió reconocer un total de 132 definidoras en el tamaño de la red y con la suma de ponderación de la frecuencia para obtener el peso semántico se reconoció que la red se conformó por 13 oraciones (tabla 4 ).

\section{Tabla 4}

Peso semántico (PS) y distancia semántica (DS) del conjunto de definidoras de la pregunta ¿qué características tienen los niños con aptitud sobresaliente en el área socioafectiva?

\begin{tabular}{lcc}
\hline \multicolumn{1}{c}{ Definidoras } & PS & DS \\
\hline Empatía & 150 & 0 \\
Regula sus emociones & 123 & 18 \\
Seguridad y confianza & 96 & 36 \\
Sensibles (emocionales) & 88 & 41 \\
Sociables & 85 & 43 \\
Es un buen líder (habilidades de liderazgo) & 71 & 52 \\
Amistosos & 59 & 60 \\
Autoestima & 58 & 61 \\
Capacidad para relacionarse con otros & 57 & 62 \\
Solidarios & 50 & 66 \\
Excelente vocabulario & 48 & 68 \\
Habilidad para convivir & 42 & 72 \\
Toma de decisiones & 41 & 72 \\
\hline
\end{tabular}

Los resultados permitieron observar que en el núcleo las docentes reportaron la palabra empatía, seguida de la idea de que los niños sobresalientes regulan sus emociones. En este sentido, Gargiulo (2011) reconoció que los niños sobresalientes en ocasiones demuestran habilidades de liderazgo. Para Deutch (2006) esta población se caracteriza por ser empáticos, sensibles, se preocupan por cuestiones éticas, buscan la perfección y se muestran autónomos e independientes, tal y como se observa en los datos reportados en este estudio. 


\section{Discusión}

La inclusión educativa de los estudiantes con distintas capacidades es un tema común e importante en la agenda nacional, que se ha retomado en diversos documentos. En estos se establece que las escuelas deben atender a todos los individuos independientemente de sus características cognitivas, físicas y emocionales. Esto ha representado un reto constante y más en algunos niveles educativos en los cuales la identificación de los niños se ha dejado de lado para priorizar otro tipo de actividades. También, se ha mencionado que el primer filtro para atender a las niñas y los niños con necesidades educativas es el docente, ya que, al estar en contacto directo con sus alumnos, provee de información valiosa que le ayuda a conocer sus características. Por este motivo, es un eje clave y el primer filtro establecido por la SEP (2020) para atender a los estudiantes con alguna necesidad específica.

Como se mencionó previamente, el docente, además de las actividades que realiza de manera cotidiana con los estudiantes en su aula, debe desarrollar una serie de competencias que le permitan el adecuado reconocimiento de las características de sus alumnos y el caso de la categoría de los niños y niñas con aptitudes sobresalientes no es la excepción. De ahí que el sistema educativo mexicano, a través de los documentos normativos, pretende regular la labor del docente para que sea capaz de reconocer las necesidades educativas de sus estudiantes para brindarles la atención adecuada y favorecer al máximo sus potencialidades.

Otro dato importante para considerar en el caso de las y los estudiantes sobresalientes es que en la Política Educativa Nacional se ha priorizado la detección en educación primaria (Covarrubias, 2012) y en una menor medida se encuentran estudios con población preescolar, aun cuando en los lineamientos de la SEP (2011) se ha marcado la trascendencia de lograr una detección temprana. En este sentido, la presente investigación pretendió profundizar sobre dicho tema y tuvo como propósito caracterizar a los niños y niñas con aptitudes sobresalientes a partir de las descripciones realizadas por las docentes de preescolar.

Con base en los resultados de esta investigación fue interesante observar que, a pesar de que las docentes de preescolar que participaron en este estudio mencionaron no haber tomado cursos sobre niños y niñas sobresalientes, fueron capaces de reconocer varias características cognitivas, creativas, motrices y socioemocionales que coinciden con 
la literatura y los estudios sobre este tema. De esta manera, se encontró que la principal característica reconocida por las docentes de preescolar fue que los niños con aptitudes sobresalientes son más imaginativos. Esta idea se ubicó en el área creativa, lo que coincide con lo reportado por Metin y Aral (2020) quienes señalaron que estos individuos tienen un elevado nivel de fantasía e ideas diferentes a las proporcionadas por sus pares.

En segundo lugar, las maestras reconocieron que los niños sobresalientes poseen una mayor coordinación motriz. Cabe mencionar que estas habilidades forman parte del repertorio de las capacidades cognitivas (Gardner, 2001; Karabulut \& Ömeroğlu, 2021) y autores como Alonso y Benito (2004) mencionan que las niñas y niños sobresaliente se desarrollan a un ritmo más rápido en el área motora, al tener un mejor control de los movimientos de su cuerpo.

Fue interesante observar que la capacidad para resolver problemas se encontró tanto en la dimensión cognitiva como en el área creativa, lo cual permite reconocer que cuando los seres humanos proporcionan soluciones a los acontecimientos cotidianos se emplean habilidades de pensamiento de orden superior. Al respecto, Sternberg et al. (2010) indicaron que en la solución de problemas participan distintos procesos que se utilizan de manera diferente en las tareas y situaciones como: inteligencia práctica (capacidad de utilizar el conocimiento en la resolución de diferentes problemas de la vida real), la creativa (ideas novedosas y originales, que implican el uso de habilidades de creación, descubrimiento e imaginación) y la analítica (uso del razonamiento y pensamiento lógico en la resolución de problemas novedosos al utilizar la experiencia previa; Rigo \& Donolo, 2013; Sternberg et al., 2010).

Otra idea que se reportó por las docentes fue que las niñas y niños sobresalientes de preescolar presentan destrezas en las cuestiones artísticas. Al respecto la SEP (2006) indicó que en la escuela se realizan varias actividades como jugar, pintar, cantar, bailar y dramatizar distintas situaciones que les permiten a los niños expresar sus emociones y sentimientos. Por lo tanto, la educación artística se propone como un área de oportunidad para desarrollar la creatividad (Chávez-Soto, 2008; Metin \& Aral, 2020; SEP, 2006).

Las siguientes características mencionadas por las docentes de preescolar se ubicaron en el área socioemocional y fueron la empatía y la regulación de emociones. Ambas ideas concuerdan con lo encontrado en la evidencia empírica, ya que se ha observado que estos estudiantes muestran capacidad para asumir las perspectivas de los otros, sensibilidad hacia las necesidades de los demás, disfrutan las relaciones sociales (Blanco, 2001; Gargiulo, 2011; López, 2008; Martín, 2004; Pfeiffer, 2008). 
Finalmente, otra de las ideas que las docentes de preescolar escribieron en mayor medida fue la capacidad de memorización, la cual se ubica en el área cognitiva. Este rasgo es uno de los que se menciona de manera repetida en la literatura, porque es una capacidad distintiva de la población sobresaliente debido al nivel de inteligencia que poseen; ellos suelen destacar en la memoria, en el procesamiento y recuperación selectiva de la información (Bergold et al., 2020; Bildiren, 2018; Lorenzo, 2006, Slater \& Howitt, 2018).

A partir de los resultados proporcionados por las docentes pareciera que las niñas y niños sobresalientes poseen un conjunto de características que les permiten alcanzar un pleno desarrollo de forma independiente; pero es necesario enfatizar que estos estudiantes requieren una intervención adecuada porque ellos tienen necesidades específicas y forman parte de un sector minoritario que los hace vulnerables (De la Torre como es citado en Reveles \& Padilla, 2018). Al respecto Del Valle (como es citado en Reveles \& Padilla, 2018) reconoce que estos niños y niñas pueden poseer altas habilidades en algunas áreas, pero presentar problemas en otras. Esto pone en evidencia la importancia de realizar evaluaciones multidimensionales en los centros escolares donde se retome al individuo, al profesor y la familia para crear programas educativos acordes con las necesidades de la población.

Se debe mencionar que una de las limitaciones en este estudio fue que la muestra solo corresponde a docentes de la Ciudad de México y pertenecen a una zona urbana, lo que las pone en cierta ventaja ante otras poblaciones. Por ello se sugiere que para futuras investigaciones se considere una muestra mayor y se incluyan maestras de preescolar de zonas vulnerables y rurales, esto a fin de conocer si los datos son similares o influyen otras variables contextuales.

Con base en los resultados del presente estudio se concluye que las docentes de preescolar que participaron en este trabajo lograron identificar diversas características vinculadas a la población sobresaliente. Por esta razón es que los docentes son uno de los informantes más comunes en el proceso de detección (Blanco, 2001). No obstante, es conveniente indicar que en ocasiones los datos de los profesores pueden ser sesgados, ya que ellos tienen estereotipos con respecto a la categoría de los sobresalientes y por este motivo distintos autores consideran que se debe complementar la información con otras pruebas formales e informales adicionales para obtener una selección más eficaz y oportuna (Chávez-Soto, 2014; Karabulut \& Ömeroğlu, 2021; Rayo, 2001).

Para garantizar que los docentes realicen una detección adecuada de las y los estudiantes sobresalientes se les deben de proporcionar cursos y talleres que les expliquen las características, mitos, estereotipos y estrategias de intervención para la categoría (Endepohls- 
Ulpe \& Ruf, 2005; Genovard, 2006; Matheis et al., 2020). Al respecto, Karabulut y Ömeroğlu (2021) y Rayo (2001) sugieren lo siguiente: preparación previa de los profesores para familiarizarlos con el proceso de identificación y con los instrumentos a emplear; contar con instrumentos adecuados para que los profesores expresen sus juicios, así como considerar a los docentes como una fuente de información más del proceso de identificación. Por lo que es necesario la combinación de otros instrumentos de evaluación, de tal forma que se establezca un perfil adecuado para cada niño y se logre un óptimo desarrollo en las diferentes esferas de su vida.

\section{Agradecimientos}

A la Escuela Berta Von Glümer por la realización de estudios de Maestría.

\section{Referencias}

Alonso, J., \& Benito, Y. (2004). Alumnos superdotados: sus necesidades educativas. Bonum. Bergold, S., Wirthwein, L., \& Steinmayr, R. (2020). Similarities and differences between intellectually gifted and average-ability students in school performance, motivation, and subjective well being. Child Quarterly, 64(4), 285-303. https://doi.org/gmjnsm

Bildiren, A. (2018). Developmental characteristics gifted children age o-6 years: Parental observations. Early Child Development and Care, 188(8), 997-1011. https://doi.org/gs7g

Blanco, V. M. C. (2001). Guía para la identificación y seguimiento de alumnos superdotados. Ciss Praxis.

Chacón, A. Y. (2005). Una revisión crítica del concepto de creatividad. Revista Electrónica Actualidades Investigativas en Educación, 5(1), 2-30.

Chávez-Soto, B. I. (2008). Programa de enriquecimiento para niños con potencial sobresaliente de segundo ciclo de primaria [Tesis de Maestría]. Universidad Nacional Autónoma de México.

Chávez-Soto, B. I. (2014). Evaluación multidimensional de alumnos con aptitud sobresaliente de educación primaria [Tesis doctoral]. Universidad Nacional Autónoma de México.

Chávez-Soto, B. I., \& Acle, T. G. (2018). Niños con altas capacidades: análisis de las variables familiares implicadas en el desarrollo del potencial. Electronic Journal of Reserach in Educational Psychology, 16(45), 273-30o. https://doi.org/10.25115/ejrep.v16i45.2094 
Chávez-Soto, B. I., Zacatelco, R. F., \& Acle, T. G. (2014). ¿Quiénes son los alumnos son aptitud sobresaliente? Análisis de diversas variables para su identificación. Actualidades Investigativas en Educación, 14(2), 1-13. https://doi.org/10.15517/aie.v14i2.14828

Covarrubias, P. P. (2012). Identificación de niñas y niños sobresalientes en preescolar [Ponencia]. Primer Congreso Internacional de Educación.

Covarrubias, P. P. (2018). Del concepto de aptitudes sobresalientes al de altas capacidades y el talento. Revista de Investigación Educativa de la REdiech, 9(17), 53-67. https:// doi.org/10.33010/ie_rie_rediech.vgi17.123

Covarrubias, P. P., \& Marín, U. R. (2015). Evaluación de la propuesta de intervención para estudiantes sobresalientes: caso Chihuahua, México. Actualidades Investigativas en Educación, 15(3), 1-32. https://doi.org/10.15517/aie.v15i3.19457

Department of Elementary \& Secondary Education. (2019). Who is the gifted learner? https://files.eric.ed.gov/fulltext/ED599012.pdf

Deutch, S. D. (2006). Bases psicopedagógicas de la educación especial. Prentice Hall.

De Zubiría, J. (2011). En contra de la visión convencional, hoy se puede afirmar que la inteligencia y el talento se desarrollan. Magisterio Editorial.

Diario Oficial de la Federación. (2011). Acuerdo número 592. https://bit.ly/zzuwzLX

Endepohls-Ulpe, M., \& Ruf, H. (2005). Primary school teacher's criteria for the identification of gifted pupils. High Ability Studies, 16(2), 219-228. https://doi.org/db4m26

Enríquez, G. A., \& Arredondo, A. (2018). Los alumnos superdotados en la agenda política educativa de México (1980-2006). Foro de Educación, 16(24), 193-213. https://doi.org/gs7j

Figueroa, J. G., González, P., \& Soliz, G. (1981). Estudios de redes semánticas naturales y algunos procesos básicos. Universidad Nacional Autónoma de México.

Gagné. F. (2012). Construyendo el talento a partir de la dotación: breve revisión del MDDT 2.o. En S. M. D. Valadez, M. J. Betancourt, \& B. M. A. Zavala (Eds.), Alumnos superdotados y talentosos: identificación, evaluación e intervención, una perspectiva para docentes (pp. 45-54). Manual Moderno.

Gardner, H. (2001). La inteligencia reformulada: las inteligencias múltiples en el siglo XXI. Paidós. Gargiulo, R. (2011). Special education in contemporary society. Sage.

Genovard, C. (2006). Educación especial para profesores de educación especial de niños excepcionales superdotados: inventando el futuro. Educar, (4), 101-110.

González-Arreola, M. R., \& Chávez-Soto, B. I. (2021). Enriquecimiento de las habilidades cognitivas de niños con aptitud sobresaliente. Revista Virtual Universidad Católica del Norte, (64), 65-9o. https://doi.org/10.35575/rvucn.n64a4 
González, J., Núñez, J., González, S., Álvarez, L., Roces, C., González, P., Bernardo, A., Valle, A., Cabanach, R., Rodríguez, S., Sales, P. (2004). Estilos de pensamiento: análisis de su validez estructural a través de las respuestas de adolescentes al Thinking Styles Inventory. Psicothema, 16(1), 139-148.

Hong, E., Greene, M. T., \& Higgins, K. (2009). Instructional practices of teachers in general education classrooms and gifted resource rooms: Development and validation of the instructional practice questionnaire. Gifted Child Quarterly, 50(2), 91-103. https:// doi.org/10.1177/001698620605000202

Karabulut, R., \& Ömeroğlu, E. (2021). A validity and reliability of a nomination scale for identifying gifted children in early childhood. International Journal of Curriculum and Instruction, 13(2), 1756-1777.

Kerlinger, F., \& Lee, H. (2002). Investigación del comportamiento. McGraw-Hill.

López, C. M. A. (2008). Estudio, mito y realidad del niño sobredotado. Trillas.

Lorenzo, R. (2006). ¿A qué se le denomina talento? Estado de arte acerca de la conceptualización. Revista Intangible Capital, 11(2), 72-153.

Manzano, A., Arranz, E., \& Sánchez de Miguel, M. (2010). Multi-criteria identification of gifted children in a Spanish sample. European Journal of Education and Psychology, 3(1), 5-17.

Martín, L. M. P. (2004). Niños inteligentes. Palabra.

Matheis, S., Keller, L. K., Kronborg, L., Schmitt, M., \& Precket, F. (2020). Do stereotypes strike twice? Giftedness and gender stereotypes in pre-service teacher's beliefs about student characteristics in Australia. Journal of Teacher Education, 48(2), 213-232. https://doi.org/10.1080/1359866x.2019.1576029

Metin, S., \& Aral, N. (2020). The drawing development characteristics gifted and children of normal development. Cypriot Journal of Educational Science, 15(1), 73-84. https:// doi.org/10.18844/cjes.v15i1.4498

Páez, F. V. E. \& Valladares, I. A. M. (2015). Detección de alumnos y alumnas con aptitudes sobresalientes en segundo y tercero de preescolar [Tesis de licenciatura]. Universidad Pedagógica Nacional, México.

Palacios, D. A. (2018). Aptitudes sobresalientes: un reto para los docentes. En J. A. Trujillo, A. Ríos, \& J. L. García (Coords.), Desarrollo profesional docente: reflexiones y experiencias de inclusión en el aula (pp. 185-195). Escuela Normal Superior Profr. José E. Medrano R. Pfeiffer, S. I. (2008). Handbook of giftedness in children. Springer. 
Piirto, J., \& Fraas, J. (2012). A mixed-methods comparison of vocational and identified gifted high school students on the Overexcitability Questionnaire. Journal for the Education of the Gifted, 35(1), 3-34. https://doi.org/10.1177/0162353211433792

Rayo, L. J. (2001). Quiénes y cómo son los superdotados. EOS

Renzulli, J. S. (2011). Theories, actions, and change: An academic journey in search of finding and developing high potential in young people. Gifted Child Quarterly, $55(4)$, 305-308. https://doi.org/10.1177/0016986211421875

Renzulli, J. S. (2012). Reexamining the role of gifted education and talent development for the 21st Century a four-part theoretical approach. Gifted Child Quarterly, $56(3)$, 150-159. https://doi.org/10.1177/0016986212444901

Reyes-Lagunes, I. L. (1993). Las redes semánticas naturales, su conceptuación y su utilización en la construcción de instrumentos. Revista de Psicología Social y Personalidad, 9(8), 81-97.

Reveles, C., \& Padilla, L. (2018, 24 de septiembre). Bullying a la inteligencia: la discriminación a niños sobresalientes en México. Animal político. https://bit.ly/zzuLIo1

Rigo, D., \& Donolo, D. (2013). Tres enfoques sobre inteligencia: un estudio con trabajadores manuales. Estudios de Psicología, 3o(1), 39-48. https://doi.org/gs7s

Rodríguez, N. E., Díaz, H. M., Rodríguez, D. M., Borges, R. A., \& Valadez, S. M. D. (2015). Programa integral para altas capacidades. Descubriéndonos: una guía práctica de aplicación. Manual Moderno.

Schuur, J., van Weerdenburg, M., Hoogeveen, L., \& Kroerbergen, E. (2021). Social-emotional characteristics and adjustment of accelerated university students: A systematic review. Gifted Child Quarterly, 65(1), 29-51. https://oi.org/10.1177/0016986220969392

Secretaría de Educación Pública. (2006). Propuesta de Intervención: atención educativa a alumnos y alumnas con aptitudes sobresalientes. Autor.

Secretaría de Educación Pública. (2011). Lineamientos para la acreditación, promoción y certificación anticipada de alumnos con aptitudes sobresalientes en educación básica. Autor.

Secretaría de Educación Pública. (2020). Principales cifras del sistema educativo nacional. Autor. Senado de la República. (2016, 11 de mayo). Gaceta de la Comisión Permanente. https:// bit.ly/3t1OR $4 \mathrm{~J}$

Slater, E., \& Howitt, C. (2018). Teacher perceptions of a pilot process for identifying intellectually gifted 6-and 7-year old children in the classroom. Australasian Journal of Gifted Educational, 32(2), 99-119. https://doi.org/10.21505/ajge.2018.0002 
Sternberg, R., Grigorenko, E., Ferrando, M., Hernández, D., Ferrándiz, C., \& Bermejo, R. (2010). Enseñanza de la inteligencia exitosa para alumnos superdotados y talentos. Revista Electrónica Interuniversitaria de Formación del Profesorado, 13(1), 111-118.

Torrance, P. (1977). Educación y capacidad creativa. Prentice Hall.

Torrance, P. (2008). Research review for the Torrance test of creative thinking figural and verbal forms $A$ and B. Scholastic Testing Service.

Valadez, S. M., Meda, L. R., \& Zambrano, G. R. (2005). Identificación de niños sobresalientes que estudian en escuelas públicas. Revista Educación y Desarrollo, (5), 39-45.

Valadez, S. M., Zambrano, G. R., \& Zavala, A. (2019). Conocimiento de los profesores de aula y de apoyo en educación básica respecto a la definición de alumnos con aptitudes sobresalientes: un estudio cualitativo. Revista Talento, Inteligencia y Creatividad, 5(10), $36-48$.

Zavala, B. M. A. (2004). La detección de alumnos CAS-Superdotados en las escuelas primarias [Tesis doctoral]. Universidad Autónoma de Aguascalientes. 